\title{
THE USE OF 3D PRE-STACK DEPTH IMAGING TO ESTIMATE LAYER VELOCITIES AND REFLECTOR POSITIONS
}

\section{INTRODUCTION}

In the case where subsurface structures are complex, it is recognized that correct depth imaging should be carried out with prestack data. When 3-D data are available, the implementation of a 3-D prestack depth migration is required. The quality of depth images heavily depends on the accuracy of the interval velocity function. Taking into account the computational effort associated with the migration, the problem of defining the interval velocity model must be solved prior to the execution of the migration task. Experience gained from 2-D work shows that structural imaging can be effectively used as a tool for interval velocity analysis in complex areas. This study tries to implement similar procedure in the 3-D case.

When 3-D prestack imaging is considered, the most important decision to make is do we really need it. In many situations, 2-D prestack depth imaging along carefully selected lines, use of well data, 3-D post-stack imaging tools and good interpretation, may be enough to construct the subsurface model. The strategy suggested here is aimed at complicated 3-D surveys where time processing fails, and should be implemented after more simplified velocity analysis and imaging techniques have already been exhausted. For practical reasons it is a robust technique that requires interpretative analysis of the results. The method will be demonstrated with some data examples.

\section{FROM 2-D TO 3-D}

There are many problem and restrictions related to the conversion of 2-D imaging techniques to 3-D. Most of the problems are technical and depend on the size of the dataset and the hardware limitations. 
For complex structures, velocity analysis by prestack imaging is an iterative procedure. The chosen imaging algorithm must be fast and flexible enough to allow the iterations. Some of the migration algorithms that require special ordering of the data (commonoffset and S-G-W migrations for example) are inadequate for a real 3-D land surveys. Accurate depth extrapolation techniques are still too expensive for today's state-ofthe-art computer systems, and even the (relatively) simple Kirchhoff-type algorithms need special computational procedures.

The basic concept of most focusing analysis techniques (Yilmaz and Chambers, 1984, Faye and Jeannot, 1986, Al-Yahya, 1989) is that after migrating prestack data with the correct velocity function, a panel collected at a given surface location should consist of flat events. This assumption is even stronger in the 3-D case. The problem of analyzing out-of-plane events, flattening and imaging them with a wrong 2-D velocity will not exist.

Directional velocity errors will result in a peculiar looking panels, and the correction needed to flatten them might not be trivial. As known from 2-D studies, conventional focusing analysis techniques are incorrect when the small-dip approximation is violated. Furthermore, when data quality is poor (low $\mathrm{S} / \mathrm{N}$ ratio, low fold), events can hardly be detected on common-surface-location panels. Any analysis of these panels may become impossible. Usually, data that requires prestack 3-D imaging suffers from at least one of the above.

\section{THE IMAGING ALGORITHM}

The imaging algorithm is based on a direct solution of the 3-D eikonal equation (Reshef, 1991). It is a shot-profile depth migration that handles irregular shooting geometries and operates from surface topography. Direct travel-time values are calculated first, and the imaging is performed in a separate stage. The algorithm can be applied to limited portions of the global output image and can be restarted from a given depth level. Storage of travel-time functions is simple and load balancing (between $\mathrm{Y} / \mathrm{O}$ and CPU time) can be optimized easily for a given hardware 
configuration.

Imaging and the following velocity analysis procedure, may be performed along arbitrary lines crossing the survey area. The cost of the procedure in this case will be highly reduced. In many situations an enhanced 2-D image will be produced by moving in data from out-of-plane by 3-D imaging. The ability to image only a portion of the survey area makes the algorithm highly suitable for target oriented migration.

\section{CVHS - CONSTANT-VELOCITY-HALF-SPACE ANALYSIS.}

This technique is an iterative "top-down" procedure for macro-model interval velocity analysis. When the velocity of a given layer is searched, it is assumed that all the velocities and reflector geometries above are correct. From the last interface downwards, the velocity is assigned to be constant. Prestack imaging is performed in this constant-velocity half space with several velocities. For each velocity, a commonsurface-location panel and a local depth image, around the analyzed location, are generated. The panels and depth-stacks differ only in the lower portion. The chosen velocity is the one that produces the "flattest" event on the panel, or, when panels are difficult to analyze, the "sharpest" image. Lateral velocity variations will be detected by performing the procedure in different locations across the layer.

The analysis is performed around a given surface location. The amount of contributing data, decimation and extent of the imaging grid and decimation of the travel-time tables, are defined according to the shooting geometry, data quality and estimated location of the area of interest.

In practice, the depth-stacks are easy to use because they have a much better $\mathrm{S} / \mathrm{N}$ ratio and give a "local" representation of the target reflector. The layer velocity is searched here with no assumption on its bottom location. The correct interval velocity, usually produces the best image which puts the reflector in its right position. Since there is no mathematical measure of the correctness of the best image, interpretation is essential part of this method. To reduce the computational effort and mainly the interpretation 
through the panel location. If 3-D imaging is used and the two depth sections for the best velocity tie well, most likely this is the layer velocity.

\section{REFERENCES}

Al-Yahya K.M, 1989, Velocity analysis by iterative profile migration, Geophysics, v. $54,718-729$.

Faye J.P., and Jeannot J.P., 1986, Prestack migration velocities from focusing depth analysis, Paper presented at the 56th Annual meeting, SEG.

Reshef M., 1991, Prestack depth imaging of three-dimensional shot gathers, Geophysics, v. $56,1158-1163$.

Yilmaz O., and Chambers R., 1984, Migration velocity analysis by wave- field extrapolation, Geophysics, v. 49, 1664-1674. 